\section{Hiperinsulinemia y resistencia insulínica en niños de dos escuelas públicas de Oaxaca, México}

\author{
ANA BEATRIZ ROSAS-SUMANO ${ }^{1}$, \\ FRANCISCO JAVIER RODAL-CANALES ${ }^{1}$, \\ MARGARITA BARRIENTOS PÉREZ ${ }^{2}$, \\ BEATRIZ EUGENIA CÁRDENAS-MORALES ${ }^{1, a}$, \\ LAURA PÉREZ-CAMPOS MAYORAL ${ }^{1, a}$, EDUARDO PÉREZ-CAMPOS ${ }^{1,3, a}$
}

\section{Hyperinsulinemia and insulin resistance in children}

Background: Obesity during childhood is a risk factor for developing cardiovascular diseases during adulthood. Aim: To measure insulin and glucose levels and parameters of insulin resistance in obese, overweight and normal weight Mexican children. Material and Methods: Comparative study of 21 obese children with a body mass index (BMI) over percentile 95, aged $10 \pm 1$ years (10 males), 14 children aged $10 \pm 2$ ( 7 males) with a BMI between percentiles 85 and 94 and 16 children aged $9 \pm 2$ years ( 3 males) with a body mass index between percentiles 10 and 84 . Body weight, blood pressure and waist circumference were measured and a blood sample was obtained to measure fasting glucose and insulin levels. Homeostasis model of insulin resistance (HOMA) and quantitative insulin sensitivity check index (QUICKI) were calculated. Results: Among obese, overweight and normal weight children, insulin levels were 14.9 (95\% CI 10.90-18.99), 7.20 (CI 5.12-9.28) and 4.73 (CI 95\% 1.92-7.53) uU/ml, respectively. The figures for HOMA were 3.16 (95\% CI 2.20-4.12), 1.49 (95\% CI 1.03-1.94) and 0.97 (95\% CI 0.35-1.60), respectively. The figures for QUICKI were 0.331 (95\% CI 0.319-0.343), 0.371 (95\% CI 0.349-0.393) and 0.419 (95\% CI 0.391-0.446), respectively. Compared to their normal weight counterparts, the risk of obese children and those with a waist circumference over percentile 90 of having a HOMA over 3.16 was 17 and 10 times higher, respectively. BMI correlated better than waist circumference with insulin levels. Conclusions: Obese children have higher levels of insulin resistance than their normal weight counterparts.

(Rev Med Chile 2016; 144: 1029-1037)

Key words: Hyperinsulinism; Insulin Resistance; Obesity.
${ }^{1}$ Centro de investigación médica y biológica (CICIMEBIO), Facultad de Medicina. Universidad Autónoma Benito Juárez de Oaxaca. UABJO. Oaxaca, Oax., México.

${ }^{2}$ Servicio de Endocrinología Pediátrica. Hospital del niño Poblano. Puebla, México. ${ }^{3}$ Centro de Investigación Interdisciplinaria UNAM-UABJO. México

aphd.

Fuente de apoyo financiero: El Dr. Eduardo Pérez Ortega y Laboratorio de Patología Clínica "Dr. Eduardo Pérez Ortega" brindaron apoyo y asistencia en la realización de las pruebas bioquímicas, sin participar en el análisis ni en la preparación ni revisión del manuscrito.

Recibido el 21 de noviembre de 2015, aceptado el 17 de junio de 2016.

Correspondencia a: Ana Beatriz Rosas Sumano M. Bravo 113. Col Centro. Oaxaca, México CP 68000 Teléfono: [951] 5163984
I a obesidad infantil es un factor de riesgo para enfermedades crónicas no transmisibles $^{1}$, como diabetes tipo 2, dislipidemias e hipertensión arterial ${ }^{2}$, asociadas a mayor riesgo cardiovascular ${ }^{3}$. Estos padecimientos integran el diagnóstico de síndrome metabólico (SM) y tienen como factor común resistencia a la insulina (RI).
Uno de los principales componentes del SM en niños, según la Organización Mundial de la Salud (OMS), es la obesidad por índice de masa corporal $(\mathrm{IMC})^{4}$ y, según la Federación Internacional de Diabetes (FID), la obesidad abdominal ${ }^{5}$. En México, la Encuesta Nacional de Salud y Nutrición 2012 (ENSANUT) reporta en niños de 5 a 11 años 
una prevalencia de sobrepeso y obesidad de $36,9 \%$ $y$ en niñas de $32,0 \%$. Obesidad $17,4 \%$ en varones y $11,8 \%$ en mujeres 6 .

En la génesis de la obesidad infantil se plantea que hay un rol en el hallazgo de hiperinsulinemia ${ }^{7}$ en ayunas causada por señalización anormal de insulina en la transducción en hígado y músculo, con pérdida de respuesta biológica de tejidos periféricos a la acción de la insulina, llamada RI, signo pivote para integrar $\mathrm{SM}^{8}$. Otros factores asociados a RI son genéticos y étnicos (más riesgo en hispano americanos) $)^{9}$. La RI ha sido documentada desde la etapa intrauterina ${ }^{10,11}$, asociándose al peso bajo al nacimiento ${ }^{12,13}$, específicamente al retraso en el crecimiento intrauterino ${ }^{14,15}$, que al exponer al niño a un ambiente obesogénico, propicia en la edad adulta $\mathrm{SM}^{16}$. También el exceso de peso al nacimiento y diabetes materna están relacionados con obesidad y RI en el niño ${ }^{17}$.

Aún no existe un punto de corte para diagnóstico de hiperinsulinemia y RI en niños ${ }^{18}$.

El propósito del estudio fue estimar valores de insulina y marcadores de RI en niños de dos escuelas públicas de Oaxaca, México, y determinar la probabilidad de hiperinsulinemia y RI.

\section{Métodos}

Estudio observacional con 51 niños de 7 a 11 años, ambos sexos, seleccionados en forma aleatoria de un total de 510 alumnos inscritos del segundo al quinto año de primaria en dos escuelas públicas de la ciudad de Oaxaca, México, con condiciones socioeconómicas similares. Se obtuvo consentimiento informado del niño y del padre o tutor, y aprobación del Comité de Ética en Investigación de la Universidad Benito Juárez de Oaxaca. Se excluyeron a los niños con Tanner $\geq 3$ de desarrollo puberal. Las mediciones fueron realizadas por un solo investigador adiestrado, especialista en Pediatría.

Se midió peso y talla en los niños vistiendo pantalón corto y camisa deportiva, sin zapatos, utilizando báscula de pie (marca Bame-420, México) con precisión de \pm 100 g y estadímetro fijo con precisión $\pm 5 \mathrm{~mm}$; se calculó el IMC (peso/talla ${ }^{2}$ ) definiéndose IMC normal entre los percentiles 10 y 84, sobrepeso entre los percentiles 85 y el 94 y obesidad por arriba del percentil 95 , de acuerdo a las gráficas del National Center for Health Statistics de Centers for Disease Control and Prevention de los Estados Unidos de Norteamérica (CDC-NCHS) en relación a sexo y edad $(2000)^{19}$. Se midió la circunferencia de cintura (CC) utilizando cinta métrica colocada $0,1 \mathrm{~cm}$ por sobre de las crestas ilíacas, con el participante en posición de pie, al final de una espiración normal. Se consideró obesidad abdominal la CC por arriba de percentil 90 de acuerdo a sexo y edad, en base a las gráficas para niños México-Americanos ${ }^{20}$.

Se tomó la presión arterial (PA) con dos esfigmomanómetros aneroides calibrados y manguitos de dimensiones estándar recomendadas, reportándose el promedio de las dos mediciones. Las cifras de PA se interpretaron según The Fourth Report from NHBPE Working Group on Children and Adolescents ${ }^{21}$.

Se consideró hipertensión arterial la PA sistólica y/o diastólica mayor a percentil 95 de acuerdo a sexo, edad y talla. Se documentó acantosis nigricans como un marcador de $\mathrm{RI}^{22-23}$ por observación directa en cuello, axilas e ingles. Se realizó valoración Tanner para desarrollo puberal mamario en mujeres ${ }^{24}$ y genital (tamaño testicular, desarrollo del pene y vello púbico) en varones ${ }^{25}$, excluyendo los que presentaban Tanner III o mayor.

Se investigó antecedentes familiares de diabetes (padres o abuelos) y peso al nacimiento (no fue posible recabar datos de semanas de gestación al nacimiento).

A todos se les efectuó determinación en sangre venosa de glicemia en ayuno, con Vitros DT60 II Chemistry System (Kodak, Rochester, NY, USA), insulina basal con Inmulite 1000 Immunoassay Analyzer (Diagnostic Products Corporation, Llanberis Glyn Rhonwy Caernarfon, UK). Se determinó, de acuerdo al criterio de la Asociación Americana de Diabetes para las glicemias de ayuno, prediabetes con valores $\geq 100$ a $125 \mathrm{mg} / \mathrm{dl}$ y diabetes $\geq 126 \mathrm{mg} / \mathrm{dl}^{26}$.

Marcadores de RI: El índice HOMA IR (Homeostatic model assessment) fue calculado con la fórmula de insulina plasmática en ayunas $(\mu \mathrm{U} /$ $\mathrm{ml}) \mathrm{x}$ glucosa plasmática ayunas $(\mathrm{mmol} / \mathrm{L}) / 22,5$. El índice QUICKI (quantitative insulin sensitivity check index) por la fórmula: $1 /(\log$ (insulina en ayunas $\mu \mathrm{U} / \mathrm{mL}$ ) $+\log$ (glucosa en ayunas $\mathrm{mg} /$ dL). Sensibilidad a la insulina y función de célula beta pancreática: Cálculo matemático a través del programa HOMA Calculator v 2.2.2. Se consideró RI con $H O M A \geq 3,16^{27}$. 


\section{Análisis estadístico}

Se utilizó $\chi^{2}$ y prueba exacta de Fisher, para comparar proporciones y buscar diferencias en variables categóricas. En variables numéricas se investigó mediante la prueba de Kolmogorov Smirnov (K-S) distribución normal de las variables. Se utilizó para comparar medias la prueba Kruskal Wallis y Anova T3 Dunnet (en caso de 3 grupos sin homogeneidad de varianzas) y $\mathrm{t}$ de Student para muestras independientes (en caso de 2 grupos) considerando el valor de $\mathrm{p}<0,05$ como significativo. Correlación de Pearson como medida de correlación en variables numéricas. Se utilizó el programa SPSS versión 18.0.

\section{Resultados}

De los 510 niños que integraron el alumnado de segundo a quinto año de las dos escuelas donde se realizó el estudio, se incluyó en la primera etapa a la población que colaboró y obtuvo consenti- miento de sus padres o tutores. Finalmente, la población en estudio fueron 51 niños que completaron el protocolo clínico y de laboratorio, 20 masculinos y 31 femeninos, de 7 a 11 años de edad (media de 9,55 años). Se clasificaron en 3 grupos en relación al IMC: a) Eutróficos: IMC entre los percentiles 10 y 84 (16 niños); b) Sobrepeso: IMC entre percentiles 85 y 94 (14 niños) y c) Obesos: IMC percentil 95 (21 niños). Los tres grupos se compararon mediante estadística descriptiva. No se observó predominio de sobrepeso y obesidad con relación al sexo, ni relación entre obesidad y peso bajo al nacimiento. En los niños con antecedentes familiares de diabetes mellitus tipo 2 se encontró un valor de riesgo 5,1 veces mayor de presentar sobrepeso y obesidad que en los que no los tenían (OR: 5,1 IC 95\% 1,41-18,66) p < 0,05. Existió asociación importante entre obesidad y acantosis nigricans $(\mathrm{p}<0,001)$ y entre obesidad por IMC y obesidad abdominal $(\mathrm{p}<0,001)(\mathrm{Ta}-$ bla 1). Se compararon los valores de glicemia,

Tabla 1. Características epidemiológicas de los niños del estudio de hiperinsulinemia y resistencia a la insulina en niños en Oaxaca, México

\begin{tabular}{|c|c|c|c|c|c|c|}
\hline \multirow[t]{2}{*}{ Características } & & & \multicolumn{3}{|c|}{ Índice de masa corporal (percentil) } & \multirow[t]{2}{*}{ p valor ${ }^{\dagger}$} \\
\hline & & & $\begin{array}{c}840< \\
n: 16\end{array}$ & $\begin{array}{c}85 \text { a } 95 \\
n: 14\end{array}$ & $\begin{array}{c}950> \\
\text { n: } 21\end{array}$ & \\
\hline Edad años (DS)* & & & $9,06(1,56)$ & $9,93(1,81)$ & $9,67(1,19)$ & NS \\
\hline Sexo & $\begin{array}{l}\text { Masc } \\
\text { Fem }\end{array}$ & $\begin{array}{l}\mathrm{n}(\%) \\
\mathrm{n}(\%)\end{array}$ & $\begin{array}{r}3(18,8) \\
13(81,2)\end{array}$ & $\begin{array}{ll}7 & (50) \\
7 & (50)\end{array}$ & $\begin{array}{l}10(47,6) \\
11(52,4)\end{array}$ & NS \\
\hline $\begin{array}{l}\text { AHF } \\
\text { Diabetes mellitus tipo } 2\end{array}$ & $\begin{array}{l}\text { Sí } \\
\text { No }\end{array}$ & $\begin{array}{l}\mathrm{n}(\%) \\
\mathrm{n}(\%)\end{array}$ & $\begin{array}{ll}7 & (43,8) \\
9 & (56,3)\end{array}$ & $\begin{array}{lr}9 & (64,3) \\
5 & (5,7)\end{array}$ & $\begin{array}{rr}19 & (90,5) \\
2 & (9,5)\end{array}$ & 0,007 \\
\hline $\begin{array}{l}\text { AHF } \\
\text { Hipertensión arterial }\end{array}$ & $\begin{array}{l}\text { Sí } \\
\text { No }\end{array}$ & $\begin{array}{l}\mathrm{n}(\%) \\
\mathrm{n}(\%)\end{array}$ & $\begin{array}{r}3(18,8) \\
13(81,3)\end{array}$ & $\begin{array}{ll}7 & (50) \\
7 & (50)\end{array}$ & $\begin{array}{ll}11 & (52,45) \\
10 & (47,6)\end{array}$ & NS \\
\hline Peso al nacimiento & $\begin{array}{l}<2.50 \\
2.500 \\
>3.50\end{array}$ & $\begin{array}{l}0 \mathrm{~g} \\
\mathrm{a} 3.500 \mathrm{~g} \\
0 \mathrm{~g}\end{array}$ & $\begin{array}{ll}5 & (31,3) \\
7 & (43,8) \\
4 & (25,0)\end{array}$ & $\begin{array}{ll}3 & (21,4) \\
8 & (57,1) \\
3 & (21,4)\end{array}$ & $\begin{array}{ll}3 & (14,3) \\
9 & (42,9) \\
9 & (42,9)\end{array}$ & NS \\
\hline Peso (kg) & (media, & , IC 95\%) & $27,6 \quad(24,1-31,0)$ & $36,6 \quad(32,8-40,5)$ & $47,5 \quad(43,6-51,5)$ & $<0,001$ \\
\hline Talla (cm) & (media, & IC 95\%) & $129,88(124-132)$ & $133,79(129-139)$ & $138,14(135-142)$ & 0,020 \\
\hline $\mathrm{IMC}\left(\mathrm{kg} / \mathrm{m}^{2}\right)$ & (media, & IC 95\%) & $16,12(15,3-16,9)$ & $20,28(19,30-21,27)$ & $24,77(23,5-25,9)$ & $<0,001$ \\
\hline Perímetro abdominal $(\mathrm{cm})$ & ) (media, & , IC 95\%) & $59,31(56,8-62,0)$ & $70,17(66,4-73,9)$ & $79,78(77,0-83,5)$ & $<0,001$ \\
\hline PA sistólica $\quad(\mathrm{mmHg})$ & (media, & , IC 95\%) & $97,19(91,5-102,8)$ & $101,07(95,0-107,0)$ & $101,43(96,5-106,2)$ & NS \\
\hline PA diastólica $(\mathrm{mmHg})$ & (media, & , IC 95\%) & $57,50(53,3-61,6)$ & $57,50(49,4-65,5)$ & $61,81(56,8-66,7)$ & NS \\
\hline Acantosis nigricans & $\begin{array}{l}\text { Sí } \\
\text { No }\end{array}$ & $\begin{array}{l}\text { n (\%) } \\
\text { n (\%) }\end{array}$ & $\begin{array}{r}1(6,3) \\
15(93,7)\end{array}$ & $\begin{array}{r}3(21,4) \\
11(78,6)\end{array}$ & $\begin{array}{r}17(81,0) \\
4(19,0)\end{array}$ & $<0,001$ \\
\hline
\end{tabular}

*DS Desviación estándar. ${ }^{\dagger}$ Comparación de los niños con peso normal (IMC < percentil 85), sobrepeso (IMC entre percentil 85 y 95) y obesidad (IMC > percentil 95). PA: Presión arterial. 
insulinemia y marcadores de RI en los tres grupos (eutróficos, sobrepeso y obesos); no hubo diferencias significativas en relación a la glicemia, sin registrarse valores por arriba de $100 \mathrm{mg} / \mathrm{dl}$.

Los valores de insulinemia fueron mayores en obesos (media 14,94 IC 95\%; 10,90-18,99) que en sobrepeso $(7,20$ IC 95\%; 5,12-9,28) y en eutróficos $(4,73$ IC 95\%; 1,92-7,53) p < 0,001, al igual que el índice HOMA: obesos (media 3,16 IC 95\%; 2,20$4,12)$, sobrepeso ( 1,49 IC 95\%; 1,03-1,94) y eutróficos $(0,97$ IC 95\%; 0,35-1,60). El I. QUICKI menor en obesos (media 0,331 IC 95\%; 0,319-0,343) que en sobrepeso $(0,371$ IC $95 \% ; 0,349-0,393)$ y eutróficos (0,419 IC 95\%; 0,391-0,446). Se demostró, mediante la prueba no paramétrica KruskalWallis, diferencia entre los tres grupos $(\mathrm{p}<0,0001)$ encontrándose valores mayores en los obesos en relación a los niños con sobrepeso y peso normal (comparación de medias por Anova T3 Dunett) $\mathrm{p}<0,001$ (Tabla 2). Posteriormente, se analizaron los niños en dos grupos formados por la presencia o ausencia de acantosis nigricans (marcador clínico de RI) sin tomar en cuenta las medidas antropométricas. Se compararon los valores de insulina, índices HOMA y QUICKI en ambos grupos, siendo la diferencia de medias significativa ( $\mathrm{t}$ de Student para muestras independientes) $\mathrm{p}<0,001$ (Tabla 3 ).

Se obtuvieron valores de corte de esta cohorte de niños, mediante curvas ROC, utilizando como variable de estado la acantosis nigricans: INSULINA: 9,7, índice HOMA: 2,14, índice QUICKI: 0,349(Figura 1). En este estudio, el riesgo de que los obesos (IMC) y con obesidad abdominal (CC $\geq$ percentil 90) tengan RI (I. HOMA $\geq 3,16)^{25}$ fue 17 y 9,16 veces mayor que los no obesos (OR 17,8 IC 95\%; 2,01-157,7) y (OR 9,6 IC 95\%; 1,77-54,83) $\mathrm{p}<0,001$ respectivamente.

Los valores de insulinemia e índice HOMA correlacionaron directa y significativamente con el IMC (r: 0,696 y r: 0,670), con la CC (r: 0,650 y r: 0,632) y con la PA sistólica (r: 0,402 y r: 0,407). El índice QUICKI correlacionó indirecta y significativamente con el IMC ( $\mathrm{r}$ : -0,750), con la CC (r: -0,697) y con la PA sistólica ( $\mathrm{r}:-0,382)$ (Tabla 4).

\section{Discusión}

El propósito del estudio fue estimar y comparar valores de insulinemia y marcadores de RI en niños oaxaqueños de 7 a 11 años de edad. Dado que el exceso de adiposidad es un factor muy importante en el desarrollo de $\mathrm{RI}^{28}$, se clasificaron los niños en relación a su estado nutricional (eutróficos, sobrepeso y obesidad) y se determinó el riesgo de hiperinsulinemia y RI en obesos, sabiendo que el desorden en la homeostasis de la glucosa puede ser causa de la ganancia de peso y de grasa visce$\mathrm{ral}^{29,30}$. Se estudiaron niños en etapa prepuberal o iniciando desarrollo puberal, ya que durante la pubertad existe aumento en la incidencia de RI, probablemente por aumento de hormona de crecimiento e IGF $1^{31}$, pero las variaciones son mayores en etapas puberales más avanzadas. Burrows y colaboradores realizan incluso una distribución percentilar de glicemia, insulinemia, QUICKI y

Tabla 2. Comparación de valores de glicemia, insulinemia y marcadores de resistencia a la insulina en los niños en relación a su estado nutricional

\begin{tabular}{|c|c|c|c|c|c|c|c|c|}
\hline \multicolumn{2}{|l|}{$\begin{array}{l}\text { IMC } \\
\text { Analitos }\end{array}$} & \multicolumn{2}{|c|}{$\begin{array}{c}950> \\
\text { Media IC 95\% }\end{array}$} & \multicolumn{2}{|c|}{$\begin{array}{c}85-94 \\
\text { Media IC 95\% }\end{array}$} & \multicolumn{2}{|c|}{$\begin{array}{c}840< \\
\text { Media IC } 95 \%\end{array}$} & \multirow{2}{*}{$\begin{array}{c}\text { Comparación } \\
\text { medias* } \\
\text { NS }\end{array}$} \\
\hline Glicemia basal & $\mathrm{mg} / \mathrm{dl}$ & 90,38 & $87,14-93,63$ & 87,64 & $83,07-92,22$ & 81,0 & $76,40-85,60$ & \\
\hline Insulina basal & $\mathrm{Uu} / \mathrm{ml}$ & 14,94 & $10,90-18,99$ & 7,20 & $12-9,28$ & 4,73 & $1,92-7,53$ & 0,001 \\
\hline Índice de HOMA & & 3,16 & $2,20-4,12$ & 1,49 & $1,03-1,94$ & 0,97 & $0,35-1,60$ & 0,001 \\
\hline Índice de QUICKI & & 0,331 & $0,319-0,343$ & 0,371 & $0,349-0,393$ & 0,419 & $0,391-0,446$ & 0,001 \\
\hline $\begin{array}{l}\text { Sensibilidad tisular } \\
\text { a insulina }\end{array}$ & $\% \mathrm{~S}$ & 66,28 & $53,22-79,34$ & 131,25 & $91,26-171,25$ & 208,15 & $157,82-258,48$ & 0,001 \\
\hline $\begin{array}{l}\text { Función célula } \beta \\
\text { pancreática }\end{array}$ & $\% B$ & 166,58 & $145,0-188,0$ & 106,05 & $88,1-123,9$ & 86,55 & $67,2-105,8$ & 0,001 \\
\hline
\end{tabular}

* Comparación de medias con ANOVA T3 Dunnet y Kruskal Wallis para muestras independientes. 
Hiperinsulinemia y resistencia a insulina en niños mexicanos - A.B. Rosas-Sumano et al

Tabla 3. Valores de insulinemia, índice Homa e índice Quicki en niños con y sin acantosis nigricans

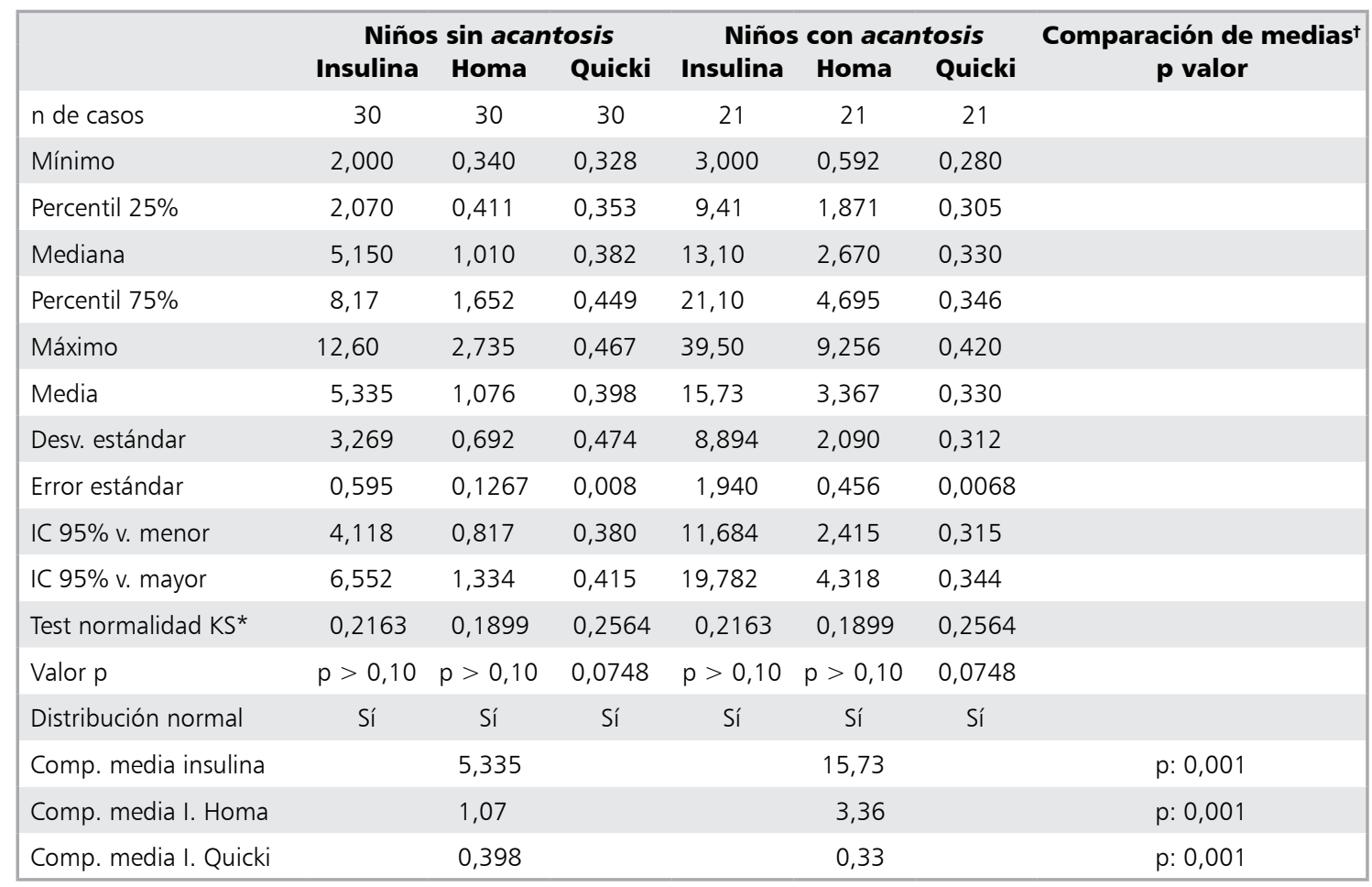

*Test normalidad de Kolmogorov Smirnov. 'Tstudents para muestras independientes.

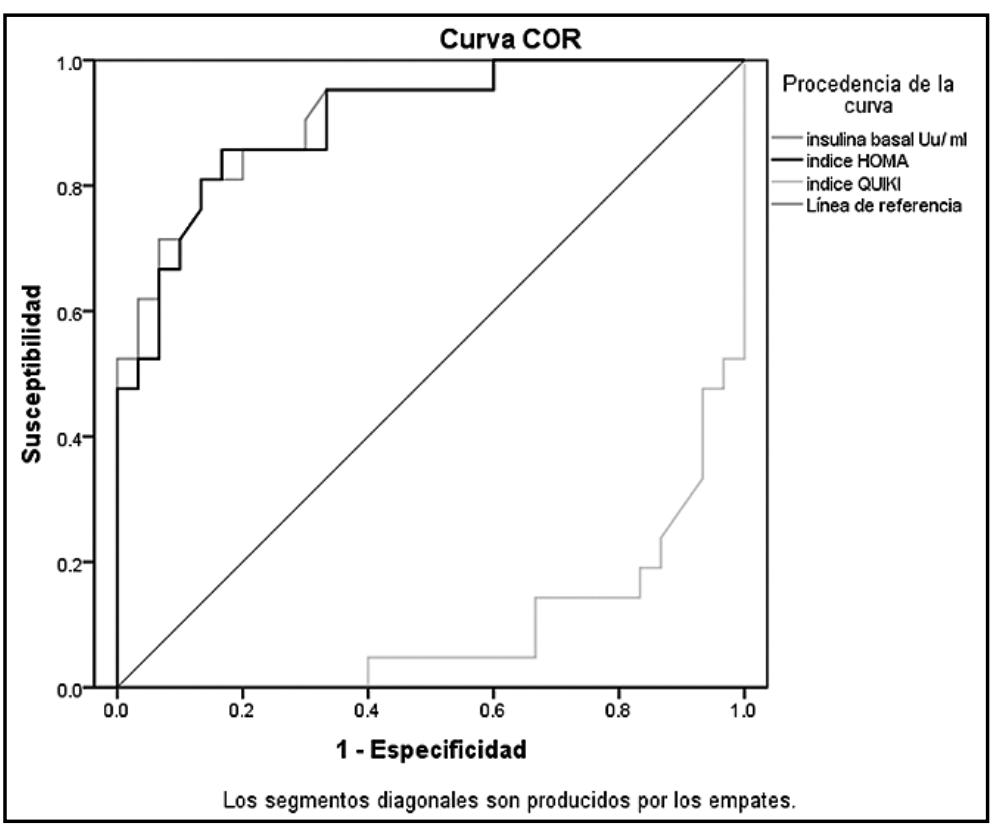

Figura 1. Curva ROC. Valores de corte de insulina, índice de Homa e índice de Quicki utilizando como variable de estado acantosis nigricans. Insulina: Área bajo la curva 0,91 (91\%) valor de corte 9,7. Índice Homa: Área bajo la curva 0,90 (90\%) valor de corte: 2,14. Índice Quicki: Área bajo la curva 0,90 (90\%) valor de corte 0,349 
Tabla 4. Correlación (r Pearson) entre insulinemia y marcadores de resistencia insulínica con variables antropométricas de los niños estudiados

\begin{tabular}{|lcc|}
\hline & $\begin{array}{c}\text { Correlación } \\
\text { de Pearson }\end{array}$ & $\begin{array}{c}\text { p } \\
\text { valor }\end{array}$ \\
Insulinemia en ayunas con: & & \\
IMC & 0,696 & 0,0001 \\
Perímetro abdominal & 0,650 & 0,0001 \\
T. A. sistólica & 0,402 & 0,003 \\
T. A. diastólica & 0,264 & $\mathrm{NS}$ \\
Índice de HOMA con: & & \\
IMC & 0,670 & 0,0001 \\
Perímetro abdominal & 0,632 & 0,0001 \\
T. A. sistólica & 0,407 & 0,003 \\
T. A. diastólica & 0,280 & 0,047 \\
Índice de QUICKI con: & & \\
IMC & $-0,750$ & 0,0001 \\
Perímetro abdominal & $-0,697$ & 0,0001 \\
T. A. sistólica & $-0,382$ & 0,006 \\
T. A. diastólica & $-0,194$ & $\mathrm{NS}$ \\
\hline
\end{tabular}

HOMA en niños de ambos sexos de 6 a 15 años formando 2 grupos: 1) Tanner I y II y 2) Tanner III- $\mathrm{V}^{32}$. Aunque en México se reporta mayor prevalencia de sobrepeso y obesidad en varones, no encontramos diferencia en relación a género, ni hubo relación entre peso bajo al nacimiento y obesidad, a diferencia de otros estudios ${ }^{12-14}$.

El encontrar en los niños con antecedentes familiares de diabetes mellitus tipo 2 una probabilidad 5,1 veces mayor de sobrepeso y obesidad, debe alertar la necesidad de realizar más estudios para investigar SM, aunque sean menores de 10 años, de acuerdo a lo propuesto por la FID 5 .

Los valores de insulinemia y marcadores de RI fueron mayores en obesos en relación a niños con sobrepeso y peso normal $(\mathrm{p}<0,001)$.

Existe mucha variabilidad en las cifras de insulina, índice HOMA e índice QUICKI en estudios en población infantil mexicana, siendo difícil determinar hiperinsulinemia y RI. Algunos autores en México ${ }^{33,34}$, sin tomar en consideración el desarrollo puberal, reportan valores mucho más elevados de insulina e índice HOMA que los obtenidos en este estudio. Otros reportan valores similares a los nuestros, con diferencias en relación al sexo no encontradas en este estudio ${ }^{35,36}$. Uno más reportó en niños obesos de 3 a 18 años, cifras de insulina muy parecidas a nuestros obesos, pero índice HOMA más elevado ${ }^{37}$.
Sin embargo, comparando nuestros resultados con los obtenidos en Chile por Burrows y $\mathrm{cols}^{32} \mathrm{en}$ 354 niños y adolescentes, clasificándolos por grado de desarrollo puberal, observamos valores de glucosa, insulina, índice HOMA e índice QUICKI en niños eutróficos y con sobrepeso de nuestro estudio, muy similares a los obtenidos por ellos en los prepúberes, no así en los obesos, pero al comparar las medias de los analitos en ambos grupos de obesos (prueba t para una muestra, utilizando como valor de la prueba las medias de insulina, HOMA y QUICKI de los niños prepúberes con obesidad de dicho estudio) existió diferencia significativa $(\mathrm{p}<0,05)$, llamando la atención que la media de IMC en los obesos en nuestro estudio fue de 24,77, cifras muy por arriba de las reportadas por Burrows en el grupo de 6 a 11 años de edad ${ }^{32}$. Dado la relación directa entre estos valores con el IMC, la diferencia que existe pudiera atribuirse a esta condición. Se ha demostrado que los niños hispano-americanos tienen mayor RI que los caucásicos, independientemente del contenido de grasa corporal, y esto puede contribuir al agotamiento y falla de las células beta pancreáticas y aparición temprana de diabetes mellitus tipo $2^{9,38}$; cada vez más frecuente, sobre todo en niñas de 10 a 19 años $^{39}$. Sin embargo, sabemos también que no todos los pacientes con obesidad tienen RI y que no todos los que cursan con RI tienen obesi$\mathrm{dad}^{18}$, por lo que se analizaron valores de glucosa, insulina, índice HOMA e índice QUICKI dejando a un lado el estado nutricional en 21 niños con acantosis nigricans, considerada como un marcador clínico de $\mathrm{RI}^{22-23}$, comparándolos con los 30 que no la presentaban, resultando la diferencia de medias entre estos dos grupos significativa $(\mathrm{p}<0,001)$ (Tabla 3$)$.

El IMC tuvo mejor correlación que la CC con la insulinemia, los índices HOMA y QUICKI. La mejor correlación fue entre IMC e índice QUICKI (r: - 0,750) p 0,001. El índice QUICKI tiene buena correlación con el clamp euglucémico hiperinsulinémico y tiene la ventaja que predice el riesgo de presentar diabetes mellitus tipo 2 mejor que el índice HOMA y la insulinemia de ayuno, lo que permite un diagnóstico más biológico de esta condición $^{40-42}$.

La OMS define como hiperinsulinémicos a las personas del quartil más alto de insulina basal de una población no diabética y en niños aún no hay consenso en dichos valores, por lo que Burrows 
y cols. sugieren en niños en etapa 1 y 2 de Tanner, hiperinsulinismo (cuartil más alto) a partir de $10 \mu \mathrm{U} / \mathrm{mL}$, el cuartil más alto de índice HOMA $\geq 2,1$ e índice QUICKI $\leq 0,34$ en dicho grupo ${ }^{32}$.

Aunque nuestra muestra es pequeña y no representativa de la población infantil oaxaqueña, los valores de corte obtenidos en nuestro estudio mediante curvas ROC: Insulina: 9,7, índice HOMA: 2,14 e índice QUICKI: 0,349 (Figura 1) son muy similares a los reportados por dichos autores, y al utilizar estos valores de referencia, encontramos que la probabilidad de que los niños obesos cursen con hiperinsulinemia es 8 veces mayor que los no obesos (OR: 8,1 IC 95\%; 2,20$29,90) \mathrm{p}<0,001$ y la probabilidad de disminución de la sensibilidad a insulina (determinada con índice QUICKI) es 9 veces mayor en los niños con obesidad (OR 9,0 IC 95\%; 2,40-33,69) p < 0,001.

Si tomamos como valor de corte para considerar RI el índice HOMA de $3,16^{25}$, el más aceptado en los niños ${ }^{43}$, que fue realizado en niños obesos con y sin RI, la probabilidad de que los obesos de este estudio cursen con RI es 17 veces mayor que en los no obesos (OR 17,8 IC 95\% 2,01-157,7) $\mathrm{p}<0,001$.

Por lo anterior, aunque este estudio es pequeño, los valores de corte reportados y que pudieran utilizarse para diagnosticar hiperinsulinemia, disminución de la sensibilidad a la insulina y RI, están por debajo de los nacionales, pero podrían ser más útiles para el diagnóstico precoz de estas alteraciones metabólicas en población como la nuestra, con antecedentes importantes de diabetes mellitus tipo 2, ya que cuando el índice HOMA sea $\geq 3,16$, la probabilidad de RI sería ya muy elevado.

\section{Conclusión}

Estos resultados indican el grave riesgo que confrontan los niños obesos estudiados en Oaxaca, México. Es necesario promover intervención temprana, mediante la detección de RI homogenizando los valores de corte, e insistiendo en la prescripción de dieta y ejercicio.

Reconocimiento: Los autores agradecen al Dr. Eduardo Pérez Ortega y al laboratorio de patología clínica "Dr. Eduardo Pérez Ortega" por su apoyo y asistencia en la realización de las pruebas bioquímicas.

\section{Referencias}

1. Kipping R, Jago R, Lawlor D. Clinical Review. Obesity in children. Part 1: Epidemiology, measurement, risk factors, and screening BMJ 2008; 337: a1824.

2. Steinberger J, Daniels SR. Obesity, insulin resistance, diabetes, and cardiovascular risk in children: an American Heart Association scientific statement from the Atherosclerosis, Hypertension, and Obesity in the Young Committee (Council on Cardiovascular Disease in the Young) and the Diabetes Committee (Council on Nutrition, Physical Activity, and Metabolism). Circulation 2003; 107: 1448-53.

3. Reaven GM. Banting Lecture 1988: role of insulin resistance in human disease. Diabetes 1988; 37: 1595-607.

4. Alberti KG, Zimmet PZ. Definition, diagnosis and classification of diabetes mellitus and its complications. Part 1. Diagnosis and classification of diabetes mellitus, provisional report of a WHO consultation. Diabet Med 1998; 15: 539-53.

5. Alberti K, Zimmet P, Shaw J. The metabolic syndrome in children and adolescents, Lancet 2007; 369: 2059-61.

6. Encuesta Nacional de Salud y Nutrición 2012. Instituto Nacional de Salud Pública. México DF.

7. Lustig R, Michele L, Mietus-Snyder M, Bacchetti P, Lazar A, Velásquez-Mieyer $\mathrm{P}$, et al. Insulin dynamics predict body mass index and Z-score response to insulin suppression or sensitization pharmacotherapy in obese children. J Pediatr 2006; 148: 23-9.

8. Raven. Insulin resistance, the insulin resistance syndrome, and cardiovascular disease. Panminerva Med 2005; 47: 201-10.

9. Goran M. Bergman R, Cruz M, Watanabe R. Insulin resistance and associated compensatory responses in African-American and Hispanic children. Diabetes Care 2002; 25: 2184-90.

10. Dunger DB, Ong KK, Huxtable SJ, Sherriff A, Woods KA, Ahmed ML, et al. Association of the INS VNTR with size at birth. ALSPAC Study Team Avon longitundinal study of pregnancy and childhood. Nat Genet 1998; 19: 98-100.

11. Stocker C, Arch J, Cawthorne M. Fetal origins of insulin resistence and obesity. Proc Nutr Soc 2005; 64: 143-51.

12. Forsen T, Eriksson J, Tuomilehto J, Reunanen A, Osmond C, Barker D. The fetal and childhood growth of persons who develop type 2 diabetes. Annals of Internal Medicine 2000; 133: 176-82.

13. Lindsay RS, Dabelea D, Roumain J, Hanson RL, Bennett $\mathrm{PH}$, Knowler WC. Type 2 diabetes and low birth weight. The role of paternal inheritance in the association of low 
birth weight andiabetes. Diabetes 2000; 49: 445-9.

14. Ong KK, Dunger DB. Birth weight, infant growth and insulin resistance. Eur J Endocrinol 2004; 151 Suppl 3: U131-9.

15. Soto N, Bazaes RA, Peña V, Salazar T, Ávila A, Iñiguez $\mathrm{G}$, et al. Insulin sensitivity and secretion are related to catch-up growth in small-for-gestational-age infants at age 1 year: results from a prospective cohort. J Clin Endocrinol Metab 2003; 88 (8): 3645-50.

16. Hales CN, Barker DJ. The thrifty phenotype hypothesis. Br Med Bull 2001; 60: 5-20.

17. James W. The epidemiology of obesity: the size of the problem. Journal Internal Medicin 2008; 263: 336-52.

18. Levy-Marchal C, Arslanian S, Cutfield W, Sinaiko A, Druet C, Marcovecchio ML, et al. ESPE-LWPESISPAD-APPES-APEG-SLEP-JSPE; Insulin Resistance in Children Consensus Conference Group. Insulin Resistance in Children: Consensus, Perspective, and Future Directions. J Clin Endocrinol Metab 2010; 95 (12): 5189-98.

19. Ogden CL, Kuczmarski RJ, Flegal KM, Mei Z, Guo S, Wei R, et al. Centers for Disease Control and Prevention 2000 growth charts for the United States: improvements to the 1977 National Center for Health Statistics version. Pediatrics 2002; 109: 45-60.

20. Fernández JR, Redden DT, Pietrobelli A, Allison DB. Waist Circumference Percentiles in nationally representative samples of African-American, European-American, and Mexican-American Children and Adolescents. J Pediatr 2004; 145: 439-44.

21. National High Blood Pressure Education Program Working Group on High Blood Pressure in Children and Adolescents. The Fourth Report on the Diagnosis, Evaluation, and Treatment of High Blood Pressure in Children and Adolescents Pediatrics 2004; 114 (2): 55576.

22. Machaca A, Saúl J. Acantosis nigricans e hiperinsulinemia en niños y adolescentes obesos del Instituto Nacional de Salud del Niño. Paediatrica 2006; 8: 64-7.

23. American Diabetes Association. Screening for Type 2 Diabetes. Diabetes Care 2003; 26: S21-S4.

24. Marshall WA, Tanner JM. Variations in pattern of pubertal changes in girls. Arch Dis Child 1969; 44 (235): 291-303.

25. Marshall WA, Tanner JM. Variations in the pattern of pubertal changes in boys. Arch Dis Child 1970; 45 (239): 13-23.

26. American Diabetes Association. Standards of medical care in diabetes 2014. Diabetes Care 2014; 37 (suppl 1): S16.

27. Keskin M, Kurtoglu S, Kendirci M, Atabek E, Yazici C.
Homeostasis model assessment is more reliable than the fasting glucose/ insulin ratio and quantitative insulin sensitivity check index for assessins insulin resistance among obese children and adolescents. Pediatrics 2005; 115: 500-3.

28. Steinberger J, Moran A, Hong CP, Jacobs DR, Jr, Sinaiko AR. Adiposity in childhood predicts obesity and insulin resistance in young adulthood. J Pediatr 2001; 138: 46973.

29. Johnson MS, Figueroa-Colon R, Huang TT, Dwyer JH, Goran MI. Longitudinal changes in body fat in African American and Caucasian children: influence of fasting insulin and insulin sensitivity. J Clin Endocrinol Metab 2001; 86: 3182-7.

30. Butte NF, Cai G, Cole SA, Wilson TA, Fisher JO, Zakeri IF, et al. Metabolic and behavioral predictors of weight gain in Hispanic children: the Viva la Familia Study. Am J Clin Nutr 2007; 85: 1478-85.

31. Moran A, Jacobs D, Steinberger J, Cohen P, Hong CP, Prineas $\mathrm{R}$, et al. Association between the insulin resistance of puberty and the insulin-like growth factor-I/ growth hormone axis. JCEM 2002; 87 (10): 4817-20.

32. Burrows R, Leiva L, Burgueño M, Maggi A, Giadrosic V, Díaz E, et al. Sensibilidad insulínica en niños de 6 a 15 años: asociación con estado nutricional y pubertad. Rev Med Chile 2006; 134: 1417-26.

33. Perichart PO, Balas NM, Schiffman SE, Barbato DA. Obesity increases metabolic syndrome risk factors in school-aged children from an urban school in Mexico City. J Am Diet Assoc 2007; 107: 81-91.

34. González-Yáñez MGE, Madero-Fernández del Castillo MA, Martínez-Ordaz VA, Serrano-Gallardo LB. Insulina, leptina y grado de resistencia a la insulina en niños escolares con y sin obesidad. Rev Esp Med Quir 2010; 15 (4): 196-203.

35. Aradillas-García C, De la Cruz-Mendoza E, Hernández $\mathrm{H}$, Calderón J, Quibrera R. Presence of insulin resistance syndrome in Mexican children of San Luis Potosí (México). Rev Salud Pública y Nutrición 2007; 8: 4.

36. Méndez-Castillo JE, Flores-Sánchez J, Noyola DE, De la Cruz-Mendoza E, Calderón-Hernández J, Aradillas-García C. Asociación del índice de resistencia a la insulina con niveles de cortisol y medidas antropométricas por género de niños mexicanos en edad escolar. Bioquimia 2007; 32 (4): 126-33.

37. Martínez-García JJ, Rojas-Pérez GG, León-Sicairos NM. Prevalencia de resistencia a la insulina y síndrome metabólico en niños obesos que acuden a la Clínica de Obesidad del Hospital Pediátrico de Sinaloa. Pediatr Mex 2010; 12 (1): 18-22.

38. Weigensberg MJ, Ball GD, Shaibi GQ, Cruz ML, Goran 
MI. Decreased beta-cell function in overweight Latino children with impaired fasting glucose. Diabetes Care 2005; 28: 2519-24.

39. Lawrence JM, Mayer-Davis EJ, Reynolds K, Beyer J, Pettitt DJ, D'Agostino RB Jr, et al. SEARCH for Diabetes in Youth Study Group. Diabetes in Hispanic American youth: prevalence, incidence, demographics, and clinical characteristics: the SEARCH for Diabetes in Youth Study. Diabetes Care 2009; 32 Suppl 2: S123-32.

40. Burrows AR, Burgueño AM, Leiva BL, Ceballos SX, Guillier OI, Gattas ZV, et al. Perfil metabólico de riesgo cardiovascular en niños y adolescentes obesos con menor sensibilidad insulínica. Rev Med Chile 2005; 133: 795-804.
41. Perseghin GC, Aumo HC, Aloni MT, Estolin G, Luzi L. Incorporation of the fasting plasma FFA con-centration into QUICKI improve its association with insulin sensitivity in nonobese individuals. JCEM 2001; 86: 4776-81.

42. Vanhala P, Vanhala M, Kumpusalo E, Keinanen-Kiukaanniemi $S$. The quantitative insulin sensitivity check index > QUICKI predicts the onset of diabetes better than fasting plasma insulin in obese subjects; a 5 years follow-up study. JCEM 2002; 87: 5834-7.

43. Martínez BA, Maldonado HJ, López AM. Métodos diagnósticos de la resistencia a la insulina en la población pediátrica. Bol Med Hosp Infant Mex 2011; 68 (5): 397-404. 